\title{
EQUIVALENT BIRATIONAL EMBEDDINGS
}

\author{
MASSIMILIANO MELLA AND ELENA POLASTRI
}

\begin{abstract}
Let $X$ be a projective variety of dimension $r$ over an algebraically closed field. It is proven that two birational embeddings of $X$ in $\mathbb{P}^{n}$, with $n \geq r+2$ are equivalent up to Cremona transformations of $\mathbb{P}^{n}$.
\end{abstract}

\section{INTRODUCTION}

Let us consider a rational variety $X \subset \mathbb{P}^{n}$. Then there exists a birational map $\phi: X \rightarrow \mathbb{P}^{r}$. The simplest embedding of $\mathbb{P}^{r}$, as a projective variety, is the linear one. It is quite natural to ask whether the map $\phi$ can be extended to a birational map $\Phi: \mathbb{P}^{n} \rightarrow \mathbb{P}^{n}$ such that $\Phi(X)$ is linear. We shall say that in this case the variety $X$ is Cremona equivalent, see Definition 1.4, to a linear space. This extension property reminds us the Abhyankar-Moh property (AMP), AM]. The latter asks about extensions of polynomial embeddings in $\mathbb{C}^{n}$ to automorphisms of $\mathbb{C}^{n}$. The AMP has been studied extensively and seems granted for high codimension smooth varieties, see for instance [Je. The AMP can be extended to affine varieties over an infinite field, say $k$. Then one can ask whether two different embeddings of the same affine variety are equivalent up to an automorphism of $k^{n}$. Also in this context the answer is positive for varieties with isolated singularities and roughly local dimension half the embedding dimension, see $[\mathrm{Sr}]$ for the precise statement.

The aim of this paper is to solve a similar question in the context of birational geometry of projective varieties. We want to understand when two birational embeddings of the same variety are equivalent up to a Cremona transformation of the projective space, in this case we say that they are Cremona equivalent, see Definition 1.4 for the precise statement. In the case of complex field this has been studied in [Je, Theorems 2.1, 2.2] where a positive answer is given for varieties of codimension greater than the dimension.

The birational nature of the problem suggests that singularities should not be a main issue. Indeed we are able to treat arbitrarily irreducible and reduced projective varieties. The main novelty comes from the range where the answer is positive. It is not difficult to give examples of rational hypersurfaces that are not Cremona equivalent to a hyperplane, see section 3 for the details. In general birationally equivalent divisors are not Cremona equivalent. What is really surprising is that this is the only case.

Theorem 1. Let $X$ be an irreducible and reduced projective variety of dimension $r$ over an algebraically closed field $k$. Then two birational embeddings in $\mathbb{P}^{n}$ are Cremona equivalent as long as $n \geq r+2$.

Date: July 2008.

1991 Mathematics Subject Classification. Primary 14E25 ; Secondary 14E08, 14N05, 14E05.

Key words and phrases. Birational maps; Cremona equivalence.

Partially supported by Progetto PRIN 2006 "Geometria sulle varietà algebriche" MUR. 
To prove the theorem we produce a chain of Cremona transformations that modify the linear systems giving the two embeddings one into the other. This is done by looking at the two birational embeddings as different projections of a common embedding. We want to stress some by-products of the main Theorem.

Corollary 1. Let $X \subset \mathbb{P}^{n}$ be a subvariety of codimension at least 2 .

If $X$ is rational then it is Cremona equivalent to a linear space.

If $X$ is birational to a smooth subvariety of $\mathbb{P}^{n}$. Then it is possible to resolve the singularities of $X$ with a Cremona transformation.

The Cremona group of $\mathbb{P}^{n}$ contains $\operatorname{Bir}(X)$, the group of birational transformations of $X$.

We like to look at the Theorem as a way to say that the Cremona group of $\mathbb{P}^{n}$ is really huge. We thank the referee for a careful reading.

\section{Notations AND PRELIMinaRies}

We work over an algebraically closed field $k$.

Definition 1.1. For any linear space $L \subset \mathbb{P}^{n}$ the map

$$
\pi_{L}: \mathbb{P}^{n} \rightarrow \mathbb{P}^{n-\operatorname{dim} L-1}
$$

is the projection from $L$.

Let $X$ be a projective irreducible and reduced variety and $\mathcal{L}$ a linear system on $X$. Assume that $\mathcal{L}$ is generated by $\left\{L_{0}, \ldots, L_{n}\right\}$. Then the map $\varphi_{\mathcal{L}}: X \rightarrow \mathbb{P}\left(\mathcal{L}^{*}\right)$ is given by evaluating the sections of $L_{i}$ at the points of $X$.

Definition 1.2. We say that $(X, \mathcal{L})$, or simply $\mathcal{L}$, is a birational embedding (in $\left.\mathbb{P}^{n}\right)$ if $\varphi_{\mathcal{L}}: X \rightarrow \mathbb{P}^{n}$ is birational onto the image. We say that $\varphi_{\mathcal{L}}=\varphi_{\mathcal{M}}$, for two birational embeddings, if there exists a dense open subset $U \subset X$ where $\varphi_{\mathcal{L}}$ and $\varphi_{\mathcal{M}}$ are both defined and have equal restriction.

Remark 1.3. Note that given a birational embedding $\mathcal{L}$ in $\mathbb{P}^{n}$ we can consider it also an embedding into $\mathbb{P}^{n+h}$ by adding $h$-times the zero section to get

$$
\mathcal{L}^{\prime}=\left\{L_{0}, \ldots, L_{n}, 0, \ldots, 0\right\}
$$

In all that follows we apply, mainly without mention, this construction to compare birational embeddings into different projective spaces.

We are interested in studying birational embeddings of a fixed variety $X$. We therefore identify $\mathcal{L}$ with $\mathcal{O}_{\varphi_{\mathcal{L}}(X)}(1)$ via $\left(\varphi_{\mathcal{L}}\right)_{*}^{-1}\left(\mathcal{O}_{\mathbb{P}^{n}}(1)\right)$.

Let $D \subset X$ be a divisor and consider the linear system $\mathcal{L}_{D}=\left\{D L_{0}, \ldots, D L_{n}\right\}$. Then we have $\varphi_{\mathcal{L}}=\varphi_{\mathcal{L}_{D}}$. In what follows we identify $\mathcal{L}$ and $\mathcal{L}_{D}$.

Definition 1.4. Let $\mathcal{L}$ in $\mathbb{P}^{n}$ and $\mathcal{G}=\left\{G_{0}, \ldots, G_{r}\right\}$ in $\mathbb{P}^{r}$ be two birational embeddings, assume that $n \geq r$ and let $\mathcal{G}^{\prime}=\left\{G_{0}, \ldots, G_{r}, 0, \ldots, 0\right\}$, obtained by adding $(n-r)$-times the zero section. We say that $\mathcal{L}$ is Cremona equivalent to $\mathcal{G}$, or simply equivalent, if there exists a birational map $\Phi: \mathbb{P}^{n} \rightarrow \mathbb{P}^{n}$ such that

$$
\varphi_{\mathcal{L}}=\Phi \circ \varphi_{\mathcal{G}^{\prime}}
$$

Such a $\Phi$ is called a (Cremona) equivalence between $\mathcal{L}$ and $\mathcal{G}$. 
Remark 1.5. The relation introduced is an equivalence relation on the birational linear systems of a fixed variety. We never ask the linear system neither to be complete nor to be minimally generated by the $L_{i}$ 's.

The equivalence $\Phi$ has to be defined on the general point of $\varphi_{\mathcal{G}^{\prime}}(X)$.

Let $\mathcal{L}$ and $\mathcal{G}$ be two linear systems on $X$. Then we have the following commutative diagram

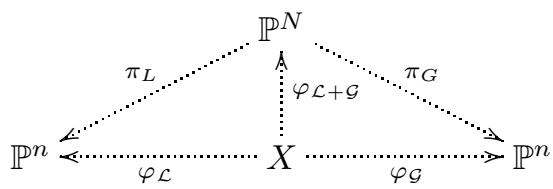

where $\mathcal{L}+\mathcal{G}=\left\{L_{i} G_{j}\right\}$, with $i, j=0, \ldots, n$, and

$$
\pi_{L} \circ \varphi_{\mathcal{L}+\mathcal{G}}=\varphi_{\mathcal{L}_{G_{0}}} \quad \pi_{G} \circ \varphi_{\mathcal{L}+\mathcal{G}}=\varphi_{\mathcal{G}_{L_{0}}}
$$

In other terms $L$ and $G$ are linear spaces spanned, respectively by $\left\{G_{j} L_{i}\right\}$, with $j=1, \ldots, n, i=0, \ldots, n$, and $\left\{G_{j} L_{i}\right\}$ with $i=1, \ldots, n, j=0, \ldots, n$.

This diagram allows us to look at two different embeddings as projections from a common one.

A natural way to construct a birational self-map of $\mathbb{P}^{n}$ is to consider a hypersurface of degree $k$ in $\mathbb{P}^{n+1}$ with two points of multiplicity exactly $k-1$. Then the projections from the singular points build up the required self-map. The following Lemma allow us to use this trick in a wide contest.

Lemma 1.6. Let $Y \subset \mathbb{P}^{n+1}$ be an irreducible reduced variety and $q_{1}, q_{2}$ two points in $\mathbb{P}^{n+1}$. Let $C Y_{i}$ be the cone over $Y$ with vertex $q_{i}$. Assume that $\operatorname{dim} Y \leq n-2$, $h^{0}\left(\mathcal{I}_{Y}(1)\right) \neq 0$ and $C Y_{i} \not \subset \operatorname{Bs}\left|\mathcal{I}_{Y}(1)\right|$. Then for $k \gg 0$ there exists an irreducible reduced hypersurface $S \in\left|\mathcal{I}_{Y}(k)\right|$ with:

- $\operatorname{mult}_{q_{i}} S=k-1$, for $i=1,2$,

- $S \not \supset C Y_{i}$.

Proof. Let $l=\left\langle q_{1}, q_{2}\right\rangle$ be the line spanned by the $q_{i}$ 's and consider the projections

$$
\pi_{q_{1}}: \mathbb{P}^{n+1} \rightarrow \mathbb{P}^{n}, \pi_{q_{2}}: \mathbb{P}^{n+1} \rightarrow \mathbb{P}^{n}, \pi_{l}: \mathbb{P}^{n+1} \rightarrow \mathbb{P}^{n-1}
$$

Let $\tilde{Y}=\pi_{l}(Y), Y_{i}=\pi_{q_{i}}(Y)$ be varieties. Then we have the following diagram

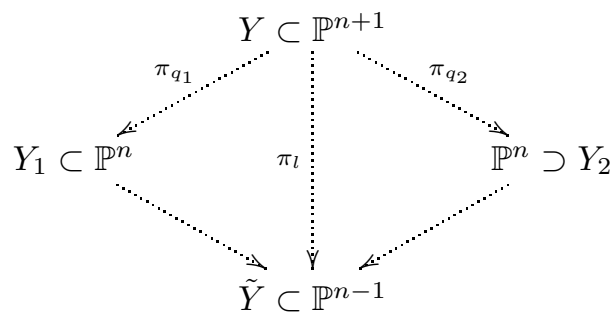

Let us consider $D=(d=0) \subset \mathbb{P}^{n-1}$ a hypersurface of degree $\delta$ with $\tilde{Y} \subset D$. Let $H=(h=0) \in\left|\mathcal{I}_{Y}(1)\right|$ be a general hyperplane. Define

$$
S=\left(d g_{1}+h g_{2}=0\right) \subset \mathbb{P}^{n+1}
$$

where:

$g_{1}$ is general of degree $k-\delta$ and multiplicity $k-\delta-1$ at $p_{i}$; 
$g_{2}$ is general with mult $q_{i} h g_{2}=k-1$.

It is easy to check that $S$ satisfies all the requirements.

\section{THE PROOF}

We are now ready to prove Theorem 1 . Let $X$ be an irreducible reduced projective variety. Let $\mathcal{L}$ and $\mathcal{G}$ be two birational embeddings in $\mathbb{P}^{n}$. Keep in mind that they are both projections of $\mathcal{L}+\mathcal{G}$ and

$$
\mathcal{L}=\mathcal{L}_{G_{0}}=\left\{L_{0} G_{0}, \ldots, L_{n} G_{0}\right\}, \quad \mathcal{G}=\mathcal{G}_{L_{0}}=\left\{L_{0} G_{0}, \ldots, L_{0} G_{n}\right\} .
$$

We want to construct a sequence of Cremona equivalent linear systems $\left\{\mathcal{A}_{i}\right\}$, for $i=0, \ldots n$, with

$$
\begin{aligned}
& -\mathcal{A}_{0}=\mathcal{L}_{G_{0}}=\left\{L_{0} G_{0}, \ldots, L_{n} G_{0}\right\} ; \\
& \text { - } \mathcal{A}_{i}=\left\{L_{0} G_{0}, \ldots, L_{0} G_{i}, A_{i+1}^{i}, \ldots, A_{n}^{i}\right\}, \text { for some } A_{j}^{i} \in|\mathcal{L}+\mathcal{G}|, j=\mathrm{i}+1, \ldots, n ; \\
& \text { - } \mathcal{A}_{n}=\mathcal{G}_{L_{0}}=\left\{L_{0} G_{0}, \ldots, L_{0} G_{n}\right\} .
\end{aligned}
$$

To prove the theorem we give a recipe that builds $\mathcal{A}_{i+1}$ from $\mathcal{A}_{i}$. Let $\mathcal{H}_{i}=$ $\left\{\mathcal{A}_{i}, L_{0} G_{i+1}\right\}$ be a linear system and $\varphi_{\mathcal{H}_{i}}: X \rightarrow \mathbb{P}\left(\mathcal{H}_{i}^{*}\right)=\mathbb{P}^{n+1}$ the associated embedding. Then we have $X_{i}=\varphi_{\mathcal{A}_{i}}(X) \subset\left(x_{n+1}=0\right) \subset \mathbb{P}^{n+1}$. Let $Z \subset \mathbb{P}^{n+1}$ be the cone over $X_{i}$ with vertex $q_{1}=[0, \ldots, 0,1]$ and $Y_{i}:=Z \cap H$ a general hyperplane section of $Z$. Then we have

$$
\pi_{q_{1}}\left(Y_{i}\right)=X_{i}
$$

and $Y_{i}$ birational to $X$. Let $q_{2} \in\left(x_{0}=\ldots=x_{i}=x_{n+1}=0\right)$ be a general point. In particular $q_{2} \notin H$ hence the projection $\pi_{q_{2} \mid Y_{i}}$ is birational onto the image. Let $X_{i+1}:=\pi_{q_{2}}\left(Y_{i}\right)$, then $X_{i+1}$ is birational to $X$ and we define $\mathcal{A}_{i+1}:=\mathcal{O}_{X_{i+1}}(1)$, keep in mind Remark 1.3. The choice of $q_{2}$ gives

$$
\mathcal{A}_{i+1}=\left\{L_{0} G_{0}, \ldots, L_{0} G_{i+1}, A_{i+2}^{i+1}, \ldots, A_{n}^{i+1}\right\}
$$

for some elements $A_{j}^{i+1} \in|\mathcal{L}+\mathcal{G}|$.

This reads in the following diagram

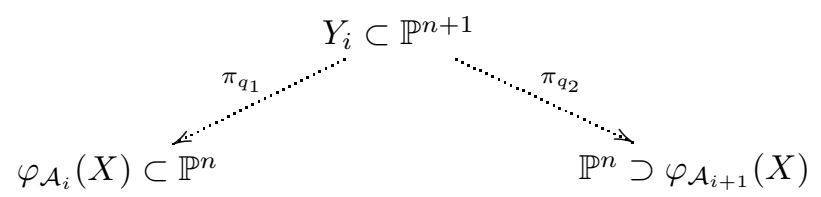

By construction $h^{0}\left(\mathcal{I}_{Y_{i}}(1)\right) \neq 0$ and $\operatorname{Bs}\left|\mathcal{I}_{Y_{i}}(1)\right| \not \supset q_{i}$, for $i=1,2$. By hypothesis we have $\operatorname{dim} Y \leq n-2$, then by Lemma 1.6 there exists an irreducible hypersurface $S \in\left|\mathcal{I}_{Y_{i}}(k)\right|$ with mult $q_{i} S=k-1$ and not containing the cones over $Y_{i}$ with vertex both $q_{1}$ and $q_{2}$. In particular

$$
\pi_{q_{1} \mid S} \text { and } \pi_{q_{2} \mid S}
$$

are birational maps to $\mathbb{P}^{n}$ and $\pi_{q_{1} \mid S}^{-1}, \pi_{q_{2} \mid S}^{-1}$ are defined on the general point of $\varphi_{\mathcal{A}_{i}}(X), \varphi_{\mathcal{A}_{i+1}}(X)$ respectively. Define the map $\Phi_{i}: \mathbb{P}^{n} \rightarrow \mathbb{P}^{n}$ as follows

$$
\Phi_{i}=\pi_{q_{2} \mid S} \circ \pi_{q_{1} \mid S}^{-1}
$$

By construction $\Phi_{i}$ is an equivalence between $\mathcal{A}_{i}$ and $\mathcal{A}_{i+1}$. 


\section{Divisorial EMBEDDINGS}

It is quite natural to expect that two divisorial embeddings are in general not Cremona equivalent. The argument of Noether-Fano inequalities gives us a way to explicitly state it for rational varieties, and therefore show that Theorem 1 is the best possible result.

Lemma 3.1. Let $X \subset \mathbb{P}^{n}$ be a rational variety of codimension 1 and degree $d>1$. Assume that $X$ is Cremona equivalent to a hyperplane. Then the singularities of the pair $\left(\mathbb{P}^{n},(n+1) / d X\right)$ are not canonical.

Proof. Let $\Phi: \mathbb{P}^{n} \rightarrow-\mathbb{P}^{n}$ be an equivalence and

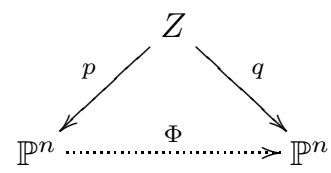

a resolution of $\Phi$. Then we have

$$
\mathcal{O}_{Z} \sim p^{*}\left(\mathcal{O}\left(K_{\mathbb{P} n}+\frac{n+1}{d} X\right)=K_{Z}+\frac{n+1}{d} X_{Z}-\sum a_{i} E_{i}\right.
$$

and

$$
q^{*}\left(\mathcal{O}\left(K_{\mathbb{P}^{n}}+\frac{n+1}{d} \mathcal{O}(1)\right)=K_{Z}+\frac{n+1}{d} X_{Z}-\sum b_{i} F_{i}\right.
$$

where $E_{i}$, respectively $F_{i}$, are $p$, respectively $q$, exceptional divisors. Let $l \subset \mathbb{P}^{n}$ be a general line in the right hand side $\mathbb{P}^{n}$. Then we have

$$
0>q^{-1} l \cdot\left(q^{*}\left(\mathcal{O}\left(K_{\mathbb{P}^{n}}+\frac{n+1}{d} \mathcal{O}(1)\right)+\sum b_{i} F_{i}\right)=\left(\sum a_{i} E_{i}\right) \cdot q^{-1} l\right.
$$

This proves that at least one $a_{i}<0$ proving the claim.

Lemma 3.1 allows to produce examples of divisor that are not Cremona equivalent to a hyperplane. Let $C \subset \mathbb{P}^{2}$ be a rational curve with only ordinary double points. If $\operatorname{deg} C \geq 6$ then $C$ is never Cremona equivalent to a line. It is easy to produce such curves by projecting a divisor of type $(1, a)$ in a smooth quadric.

One can construct similar examples in arbitrary dimension.

\section{REFERENCES}

[AM] Abhyankar, S.S., Moh, T.T.: Embeddings of the line in the plane. J. Reine Angew. Math. 276, 148-166 (1975)

[Je] Jelonek, Z.: The extension of regular and rational embeddings. Math. Ann. 277, 113-120 (1987)

[Sr] Srinivas, V.: On the embedding dimension of an affine variety. Math. Ann. 289, 125-132 (1991)

Dipartimento di Matematica, Università di Ferrara, Via Machiavelli 35, 44100 FerRARA, ITALIA

E-mail address: mll@unife.it plslne@unife.it 